\title{
TWO REMARKS ABOUT HEREDITARY ORDERS
}

\section{H. JACOBINSKI}

Abstract. In the first remark it is shown that, over a Dedekind ring, hereditary orders in a separable algebra are precisely the "maximal" orders under a relation stronger than inclusion (Theorem 1). At the same time simple proofs for known structure theorems of hereditary orders are obtained. In the second remark a complete classification is given of lattices over a hereditary order, provided the underlying Dedekind ring is contained in an algebraic number field and the lattices satisfy the Eichler condition (Theorem 2).

Let $\mathrm{o}$ be a Dedekind ring with quotient field $k, A / k$ a separable finite-dimensional algebra over $k$ and $R$ an o-order in $A$ (i.e. a finitely generated $\mathbb{D}$-algebra in $A$, containing the identity and such that $k R=A)$. An order $R$ is hereditary, if every left ideal is a projective $R$-module. It is a classical result-apart from terminology-that maximal orders are hereditary, but the converse of this is false: there are nonmaximal hereditary orders. Our first remark is, that if inclusion is replaced by a stronger relation, hereditary orders are characterized by the property of being locally maximal everywhere under this relation. To avoid confusion, we will use the term extremal orders instead. This characterization of hereditary orders can be used to give very simple proofs of some known properties of hereditary orders, which were obtained by Harada [4] and Brumer [2]. Since Brumer [2] is not available in print, we include proofs of the main results given there.

In the complete local case, the structure of $R_{p}$-lattices is well known (Brumer [2]). The basic fact is that indecomposable $R_{p}$-lattices are in fact lattices over a maximal order containing $R_{p}$. This does not hold globally and only partial results are known in that case. Using results of an earlier paper (Jacobinski [5]), we give a complete classification of lattices over a hereditary $\mathbf{D}$-order, provided the quotient field of 0 is an algebraic number field. The local theory yields a classification of genera of $R$-lattices. Our result is that the lattices in a restricted genus are isomorphic. This means that two $R$-lattices $M$ and $N$ are

Received by the editors April 24, 1970.

AMS 1970 subject classifications. Primary 16A18, 16A14; Secondary 20C10, 16A50.

Key words and phrases. Maximal orders, hereditary orders, lattices over hereditary orders, intersection of maximal orders, endomorphism ring of the radical, genera of lattices, restricted genera. 


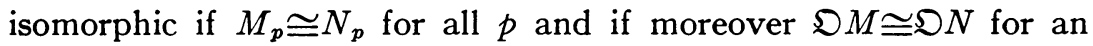
arbitrary maximal order $\mathfrak{D}$ containing $R$.

1. Let $R$ and $S$ be o-orders in $A$. We say that $S$ radically contains $R$, notation $S>R$, if $S \supset R$ and $J(S) \supset J(R)$, where $J(S), J(R)$ denotes the Jacobson radical. An order $R$ is extremal if $S>R$ implies $S=R$. Every order is radically contained in an extremal order. This follows by the same argument as the corresponding result for maximal orders (Deuring [3, p. 70]).

If $\boldsymbol{D}$ contains an infinite number of prime ideals, the Jacobson radical of every order vanishes. In this case $S>R$ reduces to $S \supset R$ and extremal orders are identical with maximal orders. Thus only a semilocal Dedekind ring $\boldsymbol{D}$ is of interest in this context and we can as well consider completions. Let $p$ be a prime in $\mathfrak{D}$ and let $\mathfrak{o}_{p}, R_{p}$ be the corresponding $p$-adic completions. Then $S_{p}>R_{p}$ is stronger than inclusion. We call an order $\mathrm{R}$ locally extremal if for each $p$, the completion $R_{p}$ is an extremal $\mathrm{o}_{p}$-order in $A_{p}$.

THEOREM 1. An D-order $R$ is hereditary if and only if it is locally extremal.

An order $R$ is hereditary if and only if $R_{p}$ is hereditary for each $p$. Thus we have to show that $R_{p}$ is hereditary if and only if it is extremal. For the proof of this, we derive first some properties of extremal orders. Let $E$ denote the right order of $J\left(R_{p}\right)$, i.e. the endomorphism ring of $J\left(R_{p}\right)$ as a left $R_{p}$-module. $E$ contains $R_{p}$ but is in general different from $R_{p}$. We first show

Proposition 1. The order $R_{p}$ is extremal if and only if $E=R_{p}$.

First we remark, that an $R_{p}$-ideal $\mathrm{I}$ is in $J\left(R_{p}\right)$ if and only if it is nilpotent modulo $p^{t} R_{p}$ for some $t>0$. The radical $J\left(R_{p}\right)$ is a right $E$ ideal, a power of which is in $p R_{p} \subset p E$. This implies $J\left(R_{p}\right) \subset J(E)$ and so $R_{p}<E$. Thus if $R_{p}$ is extremal, we must have $R_{p}=E$.

Conversely suppose $E=R_{p}$ and let $S$ be an order radically containing $R_{p}$. Then if $a$ is sufficiently large we have $p^{a} S \subset J\left(R_{p}\right)$ and so there is an integer $b$ such that $J^{b}(S) \subset J\left(R_{p}\right)$. But then $J\left(R_{p}\right) J^{b-1}(S) \subset J\left(R_{p}\right)$ and so $J^{b-1}(S)$ is contained in the endomorphism ring of $J\left(R_{p}\right)$, that is in $R_{p}$. This implies that already $J^{b-1}(S) \subset J\left(R_{p}\right)$ and by repeating this argument we obtain that $J(S)=J\left(R_{p}\right)$. Since $J(S)$ is a right $S$-ideal, this implies $S \subset R_{p}$, which completes the proof. Obviously the proposition remains valid if $E$ is replaced by the left order of $J\left(R_{p}\right)$.

Let $C_{p}$ be the maximal order in the center of $A_{p}$. Then clearly the order generated by $R_{p}$ and $C_{p}$ contains $R_{p}$ radically. If $R_{p}$ is extremal 
it must contain $C_{p}$ and is consequently a direct sum of extremal orders in simple algebras. Thus we can-and will-assume that $A_{p}$ is a simple algebra.

Now choose a maximal order $\mathfrak{D}_{p}$ that contains $R_{p}$ and denote its radical by $\mathfrak{B}$. Then $R_{p}+\mathfrak{B}>R_{p}$ and so $\mathfrak{B} \subset R_{p}$. This shows that an extremal order is completely determined by its image in $\overline{\mathfrak{D}}_{p}=\mathfrak{D}_{p} / \mathfrak{P}$.

Let $V$ be an indecomposable $\mathfrak{D}_{p}$-lattice. Its endomorphism ring $\Omega$ is then a maximal order in a skew-field and we consider $V$ as a right $\Omega$ lattice. Let $\mathfrak{p}$ be the radical of $\Omega$. Then $\bar{\Omega}=\Omega / \mathfrak{p}$ is a skew-field and $\bar{V}=V / \mathfrak{P} V$ is a right $\bar{\Omega}$-vectorspace.

Proposition 2. Let

$$
\varepsilon: \bar{V}=\bar{V}_{0} \supset \bar{V}_{1} \supset \cdots \supset \bar{V}_{s-1} \supset \bar{V}_{s}=0
$$

be a strictly descending sequence of right $\bar{\Omega}$-vectorspaces and put

$$
S(\varepsilon)=\left\{x \in \mathfrak{D}_{p}, x \bar{V}_{i} \subset \bar{V}_{i}, i=0,1, \cdots, s\right\} .
$$

Then $S(\mathcal{E})$ is an extremal $\mathrm{D}_{p}$-order and every such order $R_{p}$ can be obtained in this way by choosing a maximal order $\mathfrak{D}_{p}$ containing $R_{p}$ and defining $\&$ by $\bar{V}_{i}=J^{i} \bar{V}$, where $J$ is the radical of $R_{p}$.

First we remark that every $\bar{\Omega}$-endomorphism of $\bar{V}$ is induced by an element of $\mathfrak{D}_{p}$. Consequently, $S(\mathcal{E})$ consists of all $\mathfrak{D}$-endomorphisms $\Omega$, that take each subspace $\bar{V}_{i}$ in to itself.

The radical of $S(\mathcal{E})$ is easy to describe. It consists of all $x$ in $\mathfrak{D}_{p}$ such that $x \bar{V}_{i} \subset \bar{V}_{i+1}$. For these elements form a two-sided ideal $I$ in $S(\varepsilon)$ and $I^{s} \subset \mathfrak{B}$ implies that $I$ is in the radical. On the other hand, $S(\mathcal{E}) / I$ is isomorphic to the direct sum of the $\operatorname{End}_{\bar{\Omega}}\left(\bar{V}_{i} / \bar{V}_{i+1}\right)$, which is semisimple and so $I$ contains the radical.

Let $V_{i}$ be the inverse image of $\bar{V}_{i}$. Then $S(\varepsilon)$ can equally well be described as the set of all $x \in A_{p}$, such that $x V_{i} \subset V_{i}$ for $i=0, \cdots$, $s-1$, and the radical $J$ of $S(\varepsilon)$ as the set of all $x \in A_{p}$ such that $x V_{i} \subset V_{i+1}$ for $i=0, \cdots, s-1$. Moreover, $J V_{i}=V_{i+1}$ and so the left order $E$ of $J$ must take each $V_{i+1}$ in to itself for $i=0, \cdots, s-1$. Since $V_{s}=\mathfrak{B} V \cong V$, this means that $E=S(\varepsilon)$ and $S(E)$ is extremal according to Proposition 1.

Conversely, suppose $R_{p}$ is extremal and embed it in a maximal order $\mathfrak{D}_{p}$. Define the chain $\mathcal{E}$ by $\bar{V}_{i}=J^{i} \bar{V}$. Then clearly $S(\mathcal{E})>R_{p}$ which implies $R_{p}=S(\varepsilon)$.

We now come to the proof of the theorem. We have to show, that $R_{p}$ is hereditary if and only if it is extremal. Suppose first that $R_{p}$ is hereditary. Auslander-Goldman [1, p. 5] have shown that this implies that $J\left(R_{p}\right)$ is right invertible, i.e. $R_{p}=J U$, where $U$ 
$=\left\{x \in A_{p}, J x \subset R_{p}\right\}$. We give a different proof, using the fact that $R_{p}$-lattices have unique decomposition into indecomposable lattices. Since $R_{p}$ is hereditary, $J$ is left projective and so the indecomposable lattices occurring in $J$ are isomorphic to direct factors of $R_{p}$. Then $J$ is right invertible if every type of indecomposable lattice occurring in $R_{p}$ occurs in $J$ too. If this were not so, we could find a central idempotent $\bar{e}$ in $R_{p} / J$ such that $\bar{e} J / J^{2}=0$. Lif ting $\bar{e}$ to an idempotent $e$ of $R_{p}$, we have $e J \subset J^{2}$ and also $e J \subset e J^{2}$. Using Nakayama's lemma this means $e J=0$. Since $p e \in e J$, we see that $e=0$ and this shows that $J$ is right invertible. But the relation $J U=R_{p}$ implies that the left order of $J$ is equal to $R_{p}$. According to Proposition 1 , this implies that $R_{p}$ is extremal.

Conversely, suppose that $R_{p}$ is extremal. Then according to Proposition 2, $R_{p}=S(\varepsilon)$ where the chain $\varepsilon$ is defined by $\bar{V}_{i}=J^{i} \bar{V}$ for $i=0, \cdots, s-1$ with $J^{*} \bar{V}=0$. Since $S(\mathcal{E})$ induces all $\bar{\Omega}$-linear transformations of $\bar{V}$ that take each $\bar{V}_{i}$ into itself, it follows that $J^{s} V=\mathfrak{B} V$. If $\mathcal{E}$ is the ramification exponent of $A_{p}$ (that is $\mathfrak{P}^{\epsilon}=p \mathfrak{D}_{p}$ ), we obtain that $J^{\epsilon s} V=p V$. Put $I=p^{-1} J^{t s}$. Then $I \bar{V}_{i}=\bar{V}_{i}$ and so $I$ is a two-sided ideal in $R_{p}$. If $I$ were $\neq R_{p}$, we could find an idempotent $e$ in $R_{p}$ such that $e I \subset J$. But this implies $e \bar{V}_{i}=e I \bar{V}_{i} \subset J \bar{V}_{i}=\bar{V}_{i+1}$ and so $e$ is in the radical of $R_{p}$. This means $e=0$ and $I=R_{p}$. Now from the relation $p^{-1} J^{e s}=R_{p}$ we see that the radical $J$ is both left and right invertible. This is known to imply that $R_{p}$ is hereditary (Auslander-Goldman $[1$, p. 5]), but can easily be shown directly. Let $M$ be a left ideal in $R_{p}$. Since $J$ is invertible, $M$ and $J M$ are projective simultaneously. Replacing if necessary $M$ by $J^{-t} M$ we can assume that $M \nsubseteq J$. Via the image of $M$ in $R_{p} / J$ we obtain a decomposition

$$
M=R_{p} e \oplus M_{1}
$$

where $e$ is an idempotent and $M_{1}$ a left $R_{p}$-ideal contained in $J$. Since $R_{p} e$ is projective, we see by repetition that $M$ is projective. Thus $R_{p}$ is hereditary, which completes the proof.

By means of the theorem we can obtain very easy proofs of a number of properties of hereditary $o_{p}$-orders, which were first shown by Harada [4] and Brumer [2]. First, Proposition 2 which is now valid for hereditary orders, is identical with the main theorem of Brumer [2]. Obviously, it yields an explicit description of hereditary $\mathfrak{D}_{p^{-}}$ orders as sets of block-matrices with entries in the maximal order $\Omega$ of the underlying skew-field. We prove some additional results due to Brumer.

Proposition 3. Let $R_{p}$ be a hereditary $\mathrm{o}_{p}$-order in the separable 
algebra $A_{p}, J$ the radical of $R_{p}, \mathfrak{D}_{p}$ a maximal order containing $R_{p}, \mathfrak{B}$ its radical and $V$ an indecomposable $\mathfrak{D}_{p}$-lattice.

(a) If $\epsilon$ is the ramification index of $A_{p}$, i.e. $\mathfrak{P}^{\mathrm{e}}=p \mathfrak{D}_{p}$, then

$$
p R_{p}=J^{\epsilon s}
$$

where $s$ is the number of simple algebras in $R_{p} / J$.

(b) Every indecomposable $R_{p}$-lattice is isomorphic to a lattice $J^{i} V$ with $0 \leqq i<s$.

(c) The left order of $J^{i} V$ is a maximal order $\mathfrak{D}_{p}^{(i)}$ and

$$
R_{p}=\bigcap_{0}^{s-1} \mathfrak{D}_{p}^{(i)} .
$$

Moreover, the orders $\mathfrak{D}_{p}^{(i)}$ are the only maximal orders containing $R_{p}$.

Proof of (a). This has already been shown in the proof of the theorem.

Proof of (b). Since $R_{p}$ is hereditary, a $R_{p}$-lattice $U$ is indecomposable if and only if $A_{p} \otimes U$ is an irreducible $A_{p}$-module. Thus we can assume that $U \subset V$. Since $J$ is invertible, we can find an integer $m$ such that $J^{m} U \subset V$ but $J^{m} U \nsubseteq J V$. The image of $J^{m} U$ in $V / \mathfrak{P} V$ is then invariant under $R_{p}$ and from Proposition 2 we see that $J^{m} U=V$. Thus $J^{n} V$ represents all types of indecomposable $R_{p}$-lattices.

The left order of $J^{n} V$ is a maximal order $\mathfrak{D}_{p}^{(n)}$ since the right order is maximal. If $J^{n} V \cong J^{m} V$ as $R_{p}$-lattices, they are also isomorphic as $\mathfrak{O}_{p}^{(n)}$-lattices. From the theory of lattices over maximal orders it is well known that then $J^{n} V=J^{m} V \mathfrak{p}^{t}=J^{m} \mathfrak{B}^{t} V$. But we have seen above, that $\mathfrak{P} V=J^{s} V$. Consequently, $J^{n} V \cong J^{m} V$ if and only if $n \equiv m(s)$ which completes the proof.

Proof of (c). If the maximal order $\mathfrak{D}_{p}^{\prime}$ contains $R_{p}$, an indecomposable $\mathfrak{O}_{p}^{\prime}$-lattice is at the same time an indecomposable $R_{p}$-lattice and this implies $\mathfrak{D}_{p}^{\prime}=\mathfrak{D}_{p}^{(i)}$ according to (b). Thus the orders $\mathfrak{O}_{p}^{(i)}$ are the only maximal orders containing $R_{p}$. Clearly $\cap \mathfrak{D}_{p}^{(i)} \supset R_{p}$. On the other hand an element of this intersection takes each $J^{2} V$ into itself and is thus contained in $R_{p}$ according to Proposition 2. This completes the proof of (c).

We remark to (c) that an intersection of arbitrary maximal orders in general is not hereditary. The following corollary shows this more explicitly.

CoROLlaRy . Let $\mathfrak{D}_{p}$ be a maximal order, $\mathfrak{B}$ its radical and

$$
\mathfrak{B}=\mathfrak{D}_{s} \mathfrak{S}_{8-1} \cdots \mathfrak{D}_{1}
$$


a proper factorization of $P$, that is the left order $\mathfrak{O}_{p}^{(i)}$ of $\mathfrak{Q}_{i}$ equals the right order of $\mathfrak{Q}_{i+1}$. Then

$$
R_{p}=\bigcap_{i} \mathfrak{D}_{p}^{(i)}
$$

is hereditary and every hereditary order can be obtained in this way.

This follows immediately from Proposition 2 if we define the chain $\mathcal{E}$ by $\bar{V}=\bar{V}_{0}$ and $\bar{V}_{i}=\mathfrak{Q}_{i} \bar{V}_{i-1}$.

2. We now leave completions and consider again a Dedekind ring $\mathfrak{D}$ and a hereditary 0 -order $R$ in the separable algebra $A / k$. Some of the local results can be transferred immediately. In particular, $R$ must contain the maximal order of the center of $A$ and is thus a direct sum of hereditary orders in simple algebras. Proposition 3(c) yields that $R$ is the intersection of the maximal orders containing it. Also the Corollary can easily be globalized.

Proposition 4. Let $\mathfrak{O}$ be a maximal $\mathrm{D}$-order in $A$ and let $\mathfrak{B}_{1}, \cdots, \mathfrak{B}_{r}$ be distinct two-sided prime ideals in $\mathfrak{O}$. If

$$
\mathfrak{B}_{1} \cdots \mathfrak{P}_{r}=\mathfrak{Q}_{3} \cdots \mathfrak{Q}_{1}
$$

is a proper factorization of $\mathfrak{P}_{1} \ldots \mathfrak{B}_{r}$, i.e. the left order $\mathfrak{D}^{(i)}$ of $\mathfrak{Q}_{i}$ equals the right order of $\mathfrak{Q}_{i+1}$, then

$$
R=\bigcap_{i} \mathfrak{D}^{(i)}
$$

is hereditary and every hereditary order in A can be obtained in this way.

Since all the $\mathfrak{P}_{i}$ are distinct, the factorization above reduces at every prime $p$ to a factorization of the same type as in the Corollary. This means that each $R_{p}$ is hereditary and so $R$ is hereditary. On the other hand let a hereditary order $R$ be given and choose a maximal order $\mathfrak{O}$ containing it. According to the Corollary we have at each prime $p$ a factorization

$$
J\left(\mathfrak{O}_{p}\right)=\mathfrak{Q}_{\boldsymbol{s}_{p}}^{(p)} \cdots \mathfrak{Q}_{1}^{(p)}
$$

such that $R_{p}$ is the intersection of the left-orders of the $\mathfrak{Q}_{i}^{(p)}$. Adding if necessary some factors $\mathfrak{D}_{p}$ we can assume that the length $s_{p}$ is the same for all $p$. Define $\mathfrak{Q}_{i}$ by the condition $\left(\mathfrak{Q}_{i}\right)_{p}=\mathfrak{Q}_{i}^{(p)}$ for all $p$. Then $\mathfrak{Q}_{s} \ldots \mathfrak{Q}_{1}$ is by construction a product of distinct two-sided prime ideals of $\mathfrak{O}$. Moreover, $R$ is the intersection of the left orders of these $\mathfrak{Q}_{i}$ since this is true at each prime $p$. 
However, (b) in Proposition 3 does not hold any longer. If $M$ is an indecomposable $R$-lattice, $M_{p}$ can be decomposable and its left order is the intersection of the maximal orders corresponding to these factors, which is not maximal in general. The classification of $R$ lattices is therefore more complicated than in the complete local case. In fact only partial results seem to be known if $A$ is a direct sum of rings of matrices over commutative fields (Brumer [2]). If the quotient field $k$ of $\mathfrak{o}$ is an algebraic number field, we can give complete invariants for the types of $R$-lattices. For this we use results of Jacobinski [5], which will be quoted as GD.

Recall that two $R$-lattices belong to the same genus, notation $M \sim N$, if for all $p, M_{p} \cong N_{p}$ as $R_{p}$-lattices. In fact it is sufficient that this is true for a finite set $U$ of primes, containing all $p$ such that $R_{p}$ is not maximal (GD, Lemma 3.1).

The fact that $R_{p}$-lattices are easily classified by use of Proposition 3 (b) means that genera of $R$-lattices are equally easy to classify. The genus $\Gamma(M)$ containing $M$ is completely determined by the type of $M_{p}$ for $p \in U$. This type is determined if for each maximal order $\mathfrak{D}$ containing $R$, we know the multiplicity of each indecomposable $\mathfrak{D}_{p^{-}}$ lattice in a decomposition of $M_{p}$. For different primes $p$ in $U$, these multiplicities have to satisfy a number of relations, expressing the fact that $k_{p} \otimes M_{p} \cong k_{p} \otimes M$ (cf. [GD, Lemma 3.2]). There is no need giving these relations in detail. Since genera of $R$-lattices thus are known, our task reduces to describing the isomorphism-classes in a genus.

A $R$-lattice $M$ is said to satisfy the Eichler condition, if none of the simple algebras in $\operatorname{Hom}_{A}(k M, k M)$ is a totally definite quaternion skew-field (cf. [GD, p. 5], where this is denoted by $M \in \mathfrak{L}_{R^{\prime}}$ ). In other words, let $e_{i}$ be a primitive central idempotent in $A$ and let $e_{i} A$ be a ring of matrices over the skew-field $\Delta_{i}$. If $\Delta_{i}$ is a totally definite quaternion skew-field, $e_{i} k M$ is not allowed to be irreducible; otherwise there is no condition on $e_{i} k M$.

Let $\mathfrak{D}$ be a maximal order containing $R$. Two $R$-lattices $M, N$ are in

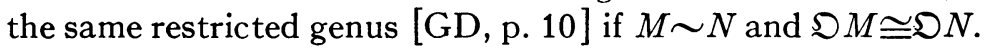

TheOREM 2. Let $\mathrm{o}$ be a Dedekind ring whose quotient field $k$ is an algebraic number field, $R$ a hereditary -order in a separable algebra $A / k$ and $M a R$-lattice, that satisfies the Eichler condition. Then all lattices in the restricted genus that contains $M$ are isomorphic or, in other words, $M \sim N$ and $\mathfrak{D} \cong \mathfrak{D} N$ implies $M \cong N$.

We can assume that $A$ is a simple algebra. According to [GD, Prop- 
osition 2.10], a lattice $N$ is in the same restricted genus as $M$ if and only if

$$
M \oplus T \cong N \oplus T
$$

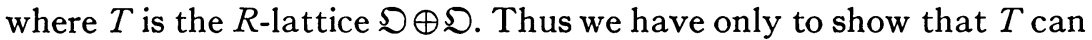
be cancelled here. According to the cancellation theorem [GD, Theorem 4.1], $T$ can certainly be cancelled if for each $p$ in $U, T_{p}$ is a direct factor of some $s M_{p}=M_{p} \oplus \cdots \oplus M_{p}$. This can be obtained easily by choosing $\mathfrak{O}$ and thus $T$ conveniently. Since $M_{p}$ is a direct sum of lattices over maximal $\boldsymbol{D}_{p}$-orders, we can choose $\mathfrak{D}$ such that $M_{p}$ contains an $\mathfrak{D}_{p}$-lattice $\neq 0$ as direct factor for each $p$ in $U$. Then clearly $\mathfrak{D}_{p}$ and also $T_{p}$ is a direct factor of $s M_{p}$ for a suitable $s$. Thus $T$ can be cancelled in $\left(^{*}\right)$, which completes the proof.

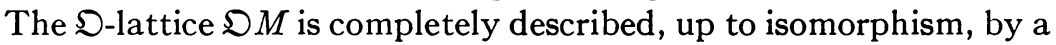
certain ideal class in the maximal order of the center of $A[\mathrm{GD}, \mathrm{p} .5]$. Thus the types of $R$-lattices are completely described by such an ideal class together with the multiplicities characterizing the genus of $M$, that we described above.

The theorem also implies the following result about cancellation.

Corollary. Let $\mathrm{D}, R$ and $M$ be as in Theorem 2 and $X$ an arbitrary $R$-lattice. Then a relation $M \oplus X \cong N \oplus X$ implies $M \cong N$.

For such a relation implies $M \sim N$ and, since $M$ satisfies Eichler's condition, also $\mathfrak{D} M \cong \mathfrak{D} N$. The theorem then yields $M \cong N$.

\section{REFERENCES}

1. M. Auslander and O. Goldman, Maximal orders, Trans. Amer. Math. Soc. 97 (1960), 1-24. MR 22 \#8034.

2. A. Brumer, Structure of hereditary orders, Thesis, Princeton University, Princeton, N. J., 1963; cf. Bull. Amer. Math. Soc. 69 (1963), 721-724; addendum, ibid. 70 (1964), 185. MR $28 \# 115$.

3. M. Deuring, Algebren, Springer, Berlin, 1934.

4. M. Harada, Hereditary orders, Trans. Amer. Math. Soc. 107 (1963), 273-290. MR 27 \#1474.

5. H. Jacobinski, Genera and decompositions of lattices over orders, Acta Math. 121 (1968), 1-29.

Chalmers University of Technology, Göteborg, Sweden 